\title{
Orientation of respiratory tract cilia in patients with primary ciliary dyskinesia, bronchiectasis, and in normal subjects
}

\author{
R DE IONGH, J RUTLAND \\ From the Respiratory Unit, Concord Hospital, Sydney, New South Wales, Australia
}

SUMMARY A reproducible technique, utilising a graphics tablet and a personal computer for measuring ciliary orientation from electron micrographs of ciliated epithelium, was assessed. Ciliary deviation was measured in 47 normal subjects (mean ciliary deviation \pm 1 SD was $14 \cdot 6\left(3 \cdot 3^{\circ}\right)$ ), in eight patients with bronchiectasis and normal ciliary function $\left(15 \cdot 1\left(6 \cdot 5^{\circ}\right)\right)$, and in seven patients with primary ciliary dyskinesia $\left(38.7\left(7 \cdot 8^{\circ}\right)\right.$; significantly greater than the first two groups). Measurements of ciliary deviation at the tip, base, and basal feet showed very little variation along the ciliary shaft in all three groups, suggesting that valid measurements of ciliary deviation can be made at any level of the cilium. Mean ciliary deviation in normal subjects was always less than $30^{\circ}$; all patients with a mean ciliary deviation of greater than $30^{\circ}$ had recurrent respiratory tract disease. Four of seven patients with primary ciliary dyskinesia had ciliary disorientation; in one this was the only defect. Measurements of inter- and intraobserver variability using this method showed a maximum difference between observations of $4 \cdot 1^{\circ}$.

It is suggested that ciliary orientation should be measured in patients suspected of having defective ciliary function or structure, or both.

Primary ciliary dyskinesia, which encompasses Kartagener's syndrome and the immotile cilia syndrome, is an autosomal recessive disorder which is characterised clinically by a history of chronic upper or lower respiratory tract infections, or both, usually evident from childhood. The respiratory tract disease is associated commonly with sinusitis, otitis media, male infertility and often dextrocardia. Ultrastructural examination usually shows one or more defects of the axoneme of cilia and spermatozoa. Reduced ciliary and sperm motility and impaired mucociliary clearance result.

Mucociliary clearance is one of the most important defence mechanisms of the respiratory tract. ${ }^{12}$ Beating cilia, which in normal subjects are oriented in one direction, move overlying mucus towards the pharynx. ${ }^{3}$ When cilia are not oriented, mucociliary clearance is grossly impaired, as in subjects with primary ciliary dyskinesia. ${ }^{45}$ Many reports of patients

Accepted for publication 2 February 1989; with primary ciliary dyskinesia have examined the defects of dynein arms and other ultrastructural defects, ${ }^{6-8}$ but few studies have quantified defects of ciliary orientation. ${ }^{9-11}$ In the absence of other ultrastructural defects ciliary disorientation alone has been reported in patients with primary ciliary dyskinesia. ${ }^{12}$

We report a simple and reproducible method for quantifying ciliary orientation in samples of ciliated epithelium, obtained non-invasively. ${ }^{13} 14$ This technique has been used successfully in the ultrastructural screening of respiratory tract cilia from normal subjects and patients with respiratory disease in our laboratory over the past three years. Ciliary orientation was studied at two ciliary levels and at the basal feet to determine whether orientation varied.

\section{Material and methods}

A study was made of 47 normal subjects (mean (SD) age 42 (4) years; range 1-74), eight patients with bronchiectasis but with normal ciliary function (mean (SD) age 29 (4); range 1-45), and seven patients with 
recurrent respiratory tract disease and abnormal ciliary function (mean (SD) age 11 (3); range 3-37). None of the normal subjects had any respiratory tract infection and no previous history of clinically important respiratory disease. Samples of ciliated epithelium were obtained by nasal mucosal brushing from the inferior nasal turbinate of each patient and the ciliary beat frequency was measured photometrically. ${ }^{13}$

Further mucosal brushings were fixed in $2.5 \%$ glutaraldehyde in $0.1 \mathrm{M}$ cacodylate buffer $(\mathrm{pH} \mathrm{7.4)}$ and $2 \mathrm{mM} \mathrm{MgSO}_{4}$ for three hours and then stored overnight in cacodylate buffer. The samples were then post-fixed in $2 \%$ osmium tetroxide, stained "en bloc" with uranyl acetate, and dehydrated in graded ethanol solutions. At the final dehydration step the specimens were treated with $1 \%$ tannic acid in $100 \%$ ethanol for 10 minutes and embedded in Spurr's resin. Suitable areas of ciliated epithelium were located in semi-thin $(1 \mu \mathrm{m})$ sections after which thin sections $(90-100 \mathrm{~nm})$ were prepared using a diamond knife on a LKB-IV ultramicrotome. The thin sections were then stained with lead citrate and examined in a Philips 201 electron microscope.

Cilia from contiguous strips of epithelium were photographed and all micrographs were enlarged to a final magnification of $\times 65000$. At this magnification central tubules can be resolved sufficiently to permit accurate determination of the ciliary beat axis (perpendicular to a line drawn through the centres of the two central tubules), at the same time allowing large numbers of cilia to be examined in each field. Ciliary angles were measured with the aid of a graphics tablet interfaced with a personal computer (Apple IIe). The computer and graphics tablet were programmed to calculate the angle $\left(0-180^{\circ}\right)$ subtended by the line through the central tubules and an arbitrary reference line (the ciliary angle). The reference line was chosen so that as many central tubule lines as possible were oriented at right angles, or nearly so, to the reference line. The line through the central tubules was delineated on the graphics tablet by two points along its length, allowing a large number of ciliary angles to be measured quickly. The mean of the ciliary angles for each field was calculated and subtracted from each measured angle resulting in a series of differences from the mean ciliary angle with values between $-90^{\circ}$ and $+90^{\circ}$. The mean of these differences is theoretically zero, and the standard deviation provides a measure of the variation in ciliary beat axis and ciliary deviation. Because the computer treats each angle as a difference from the mean ciliary angle, data from other fields in the same specimen can be pooled without the need for all fields to be aligned with each other. This is particularly useful for examining sections from the same specimen which are on different grids and for sampling groups of cilia from strips of epithelium that are not contiguous.
Photomicrographs were prepared for cilia sectioned near the base and for cilia sectioned near the tip from each sample. Microvillus processes extend about half the length of the cilia (as seen in longitudinal section), so that in transverse sections the presence of microvilli indicates a plane of section nearer the base of the cilia, whereas the absence of microvilli indicates a plane of section nearer the tips (fig 1). Where the plane of section included basal feet, measurements of ciliary deviation, defined by a line passing through the centre of the centriole and the apex of the basal foot were also made (figs 1 and 2). After the ciliary deviation in these three regions had been analysed separately, all data for each patient were pooled to provide a total or overall measure of ciliary deviation for that patient. A mean of 159 ciliary angles (range: 75-279) was measured to calculate the variation in ciliary axis in each specimen. All measurements of ciliary deviation were conducted by one observer (RDI).

The differences between ciliary deviation for the three groups were compared statistically by analysis of variance and Mann-Whitney U tests.

INTEROBSER VER VARIABILITY IN MEASUREMENTS OF CILIARY DEVIATION

To examine the interobserver variability in the

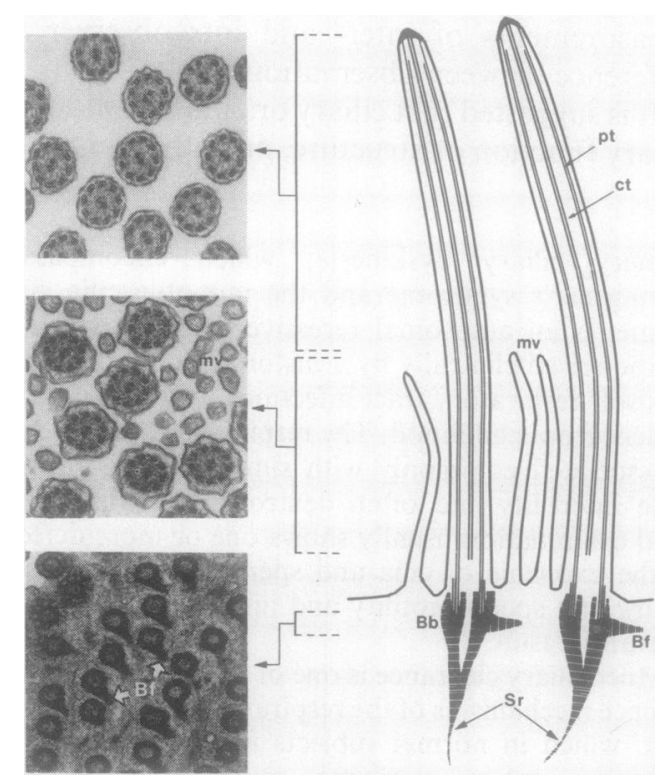

Fig 1 Diagram and representative micrographs showing the three regions of the cilium in which ciliary orientation measurements were made. Microvilli ( $m v$ ) occur near the proximal part of the ciliary axoneme. Sections through the basal body $(B b)$ permitted measurements of basal foot ( $B f)$ orientation. Sr, striated roots; pt, peripheral microtubules; $c t$, central microtubules. 

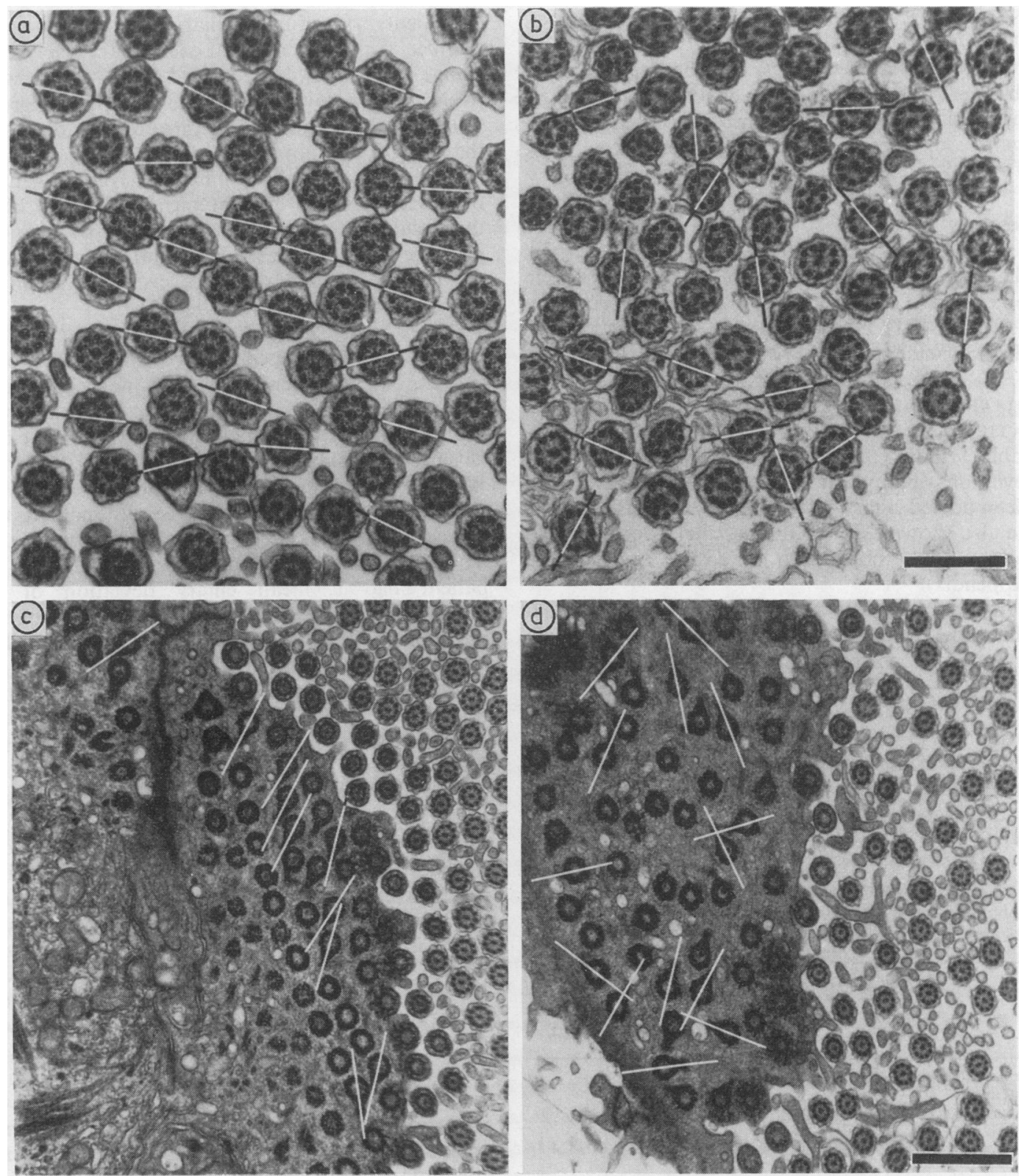

Fig 2 Micrographs showing ciliary orientation determined by a line drawn through the central tubules of cilia in: (a) normal subject, mean ciliary deviation in this field is $11 \cdot 6^{\circ}$; (b) patient with abnormal ciliary function and random ciliary orientation, mean ciliary deviation is $51.9^{\circ}$. Ciliary orientation could also be measured from the direction of basal foot projection: (c) normal subject, basal foot orientation is $16 \cdot 6^{\circ} ;(d)$ patient with abnormal ciliary function and random orientation of basal feet, 50.7०. Scale bar $a$ and $b, 0.5 \mu \mathrm{m} ; \mathrm{c}$ and d, $1.0 \mu \mathrm{m}$.

measurement of ciliary deviation triplicate copies of 20 micrographs, representing four to six fields from each of four cases (two normal subjects and two patients with" primary ciliary dyskinesia), were measured "blind" by three separate observers. Variabilities in the ciliary deviation obtained for each field and for each patient were compared by one way analysis of variance and by coefficients of variation. 
INTRAOBSER VER VARIABILITY IN MEASUREMENTS OF CILIARY DEVIATION

To assess the repeatability of the technique one observer (RDI) measured the ciliary deviation of five triplicate sets of micrographs. The variability was again assessed by analysis of variance and coefficients of variation.

\section{Results}

The rate of ciliary beat (mean \pm SD) for the normal subjects was $11.4(1.4) \mathrm{Hz}$; for patients with bronchiectasis $12.2(1 \cdot 1) \mathrm{Hz}$; and for patients with primary ciliary dyskinesia $4 \cdot 7(4 \cdot 8) \mathrm{Hz}$.

\section{CILIARY DEVIATION IN NORMAL SUBJECTS AND PATIENTS}

Table 1 shows the mean (SD) and range of ciliary deviations obtained at the three levels for each group. Mean pooled ciliary deviations for each patient group are also shown. The maximum ciliary deviation of a truly random population of ciliary angles is $52^{\circ}$ (Appendix).

In normal subjects the mean number (and range) of cilia studied at the level of the basal feet, the microvilli, and above the microvilli, was 32 (12-85), 82 (41-139), and 75(22-135), respectively. Measurements of ciliary angle in the three regions of the cilium showed that the variations in ciliary deviation were similar, mean deviations being $14-15^{\circ}$ in each region (range 6-28 $8^{\circ}$. For patients with bronchiectasis the mean number (and range) of cilia examined in the three regions were 24 (10-39), 88 (45-141), and 80 (24-138), respectively.

Table 1 Variations in ciliary deviation at three different levels along length of ciliary shaft in normal subjects and patients

\begin{tabular}{|c|c|c|c|c|}
\hline & \multicolumn{3}{|l|}{ Ciliary level } & \multirow{2}{*}{$\begin{array}{l}\text { Total } \\
\text { pooled } \\
\text { deviation }\end{array}$} \\
\hline & $\begin{array}{l}\text { Basal } \\
\text { feet }\end{array}$ & Villi & $\begin{array}{l}\text { No } \\
\text { villi }\end{array}$ & \\
\hline $\begin{array}{l}\text { Normal subjects } \\
\text { Mean } \\
\text { deviation }\left(^{\circ}\right) \\
\text { SD }\left(^{\circ}\right) \\
\text { (Range) } \\
n=\end{array}$ & $\begin{array}{l}14 \cdot 4 \\
4 \cdot 0 \\
(7 \cdot 3-21) \\
10\end{array}$ & $\begin{array}{l}14 \cdot 0 \\
3 \cdot 8 \\
(6 \cdot 3-27 \cdot 8) \\
47\end{array}$ & $\begin{array}{l}14 \cdot 8 \\
3 \cdot 5 \\
(8 \cdot 7-26 \cdot 6) \\
47\end{array}$ & $\begin{array}{l}14 \cdot 6 \\
3 \cdot 3 \\
(8 \cdot 9-24 \cdot 5) \\
47\end{array}$ \\
\hline $\begin{array}{l}\text { Bronchiectasis: } \\
\text { Mean } \\
\text { deviation }\left(^{\circ}\right) \\
\text { SD }\left(^{\circ}\right) \\
\text { (Range) } \\
n=\end{array}$ & $\begin{array}{l}16 \cdot 3 \\
1.9 \\
(14 \cdot 7-18 \cdot 4) \\
3\end{array}$ & $\begin{array}{l}12 \cdot 3 \\
3 \cdot 7 \\
(6 \cdot 2-18 \cdot 9) \\
8\end{array}$ & $\begin{array}{l}16 \cdot 2 \\
6 \cdot 2 \\
(9 \cdot 8-30 \cdot 2) \\
8\end{array}$ & $\begin{array}{l}15 \cdot 1 \\
6 \cdot 5 \\
(8 \cdot 6-30 \cdot 2) \\
8\end{array}$ \\
\hline $\begin{array}{l}\text { Primary ciliary } \\
\text { Mean } \\
\text { deviation }\left(^{\circ}\right) \\
\text { SD }\left(^{\circ}\right) \\
\text { (Range) } \\
\mathbf{n}=\end{array}$ & $\begin{array}{l}\text { dyskinesia: } \\
\qquad \begin{array}{l}47 \cdot 7 \\
4 \cdot 3 \\
(42 \cdot 7-50 \cdot 3) \\
3\end{array}\end{array}$ & $\begin{array}{l}34 \cdot 8 \\
10 \cdot 8 \\
(25 \cdot 7-49 \cdot 5) \\
7\end{array}$ & $\begin{array}{l}34 \cdot 6 \\
11 \cdot 9 \\
(21 \cdot 1-51 \cdot 8)\end{array}$ & $\begin{array}{l}38.7 \\
11.8 \\
(29.4-51.8) \\
7\end{array}$ \\
\hline
\end{tabular}

In patients with primary ciliary dyskinesia the mean number of cilia examined at each ciliary level was 28 (range 12-52), 93 (43-136), and 66 (39-140), respectively. Ciliary deviations in the two patient groups were also similar among the three regions but less so than for normal subjects. Analysis of variance showed no significant differences in ciliary deviation between the three regions of measurement within any of the three subject groups. In the three patients with primary ciliary dyskinesia in whom ciliary deviation was measured at the basal foot level, orientation was found to be almost random $\left(43^{\circ}-50^{\circ}\right)$.

Pooled ciliary deviations in normal subjects ranged from $9^{\circ}$ to $25^{\circ}$. The mean pooled ciliary deviation in patients with bronchiectasis was not significantly different from that of normal subjects. In patients with primary ciliary dyskinesia, however, the mean ciliary deviation was significantly greater ( $p<0.05$; MannWhitney $U$ test) than in both normal subjects and patients with bronchiectasis. Three of the patients with primary ciliary dyskinesia had a mean ciliary deviation within the range of normal subjects $\left(<30^{\circ}\right)$ and one patient had a mean ciliary deviation of $32^{\circ}$ (moderately disoriented). The other three patients had mean ciliary deviations of $45^{\circ}, 50^{\circ}$, and $51^{\circ}$, which from theoretical calculations (Appendix) indicate almost random orientation of cilia. The random orientation of cilia and basal feet in such patients compared with that of a normal subject is shown in fig 2 .

Fig 3 shows the pooled distribution histograms of ciliary deviations for each of the three subject groups studied. Each bar represents the percentage of cilia with deviations from the mean ciliary angle (positive or negative) in intervals of $10^{\circ}$. In normal subjects and in patients with bronchiectasis the population of angles is a normal distribution centred around zero (the mean angle) and with $95 \%$ of cilia having deviations from the mean angle of less than $30^{\circ}$ (dotted line). In the pooled distribution for the patients with ciliary dyskinesia $95 \%$ of cilia had deviations between $0^{\circ}$ and $80^{\circ}$. If cilia are oriented randomly the distribution should be uniform (Appendix). Two patients with primary ciliary dyskinesia, however, had near normal orientation (albeit reduced) and two had normal orientation, producing the distribution shown in fig 3. In some normal subjects there were cilia with large deviations from the mean angle $\left(>30^{\circ}\right)$. These were few, and their effect on mean ciliary deviation was minimal.

\section{INTEROBSER VER VARIABILITY}

Table 2 shows the mean (SD) ciliary deviations, the coefficients of variation, and the maximum differences in degrees between the ciliary deviation obtained for each patient by the three observers. In cases 3 and 4 

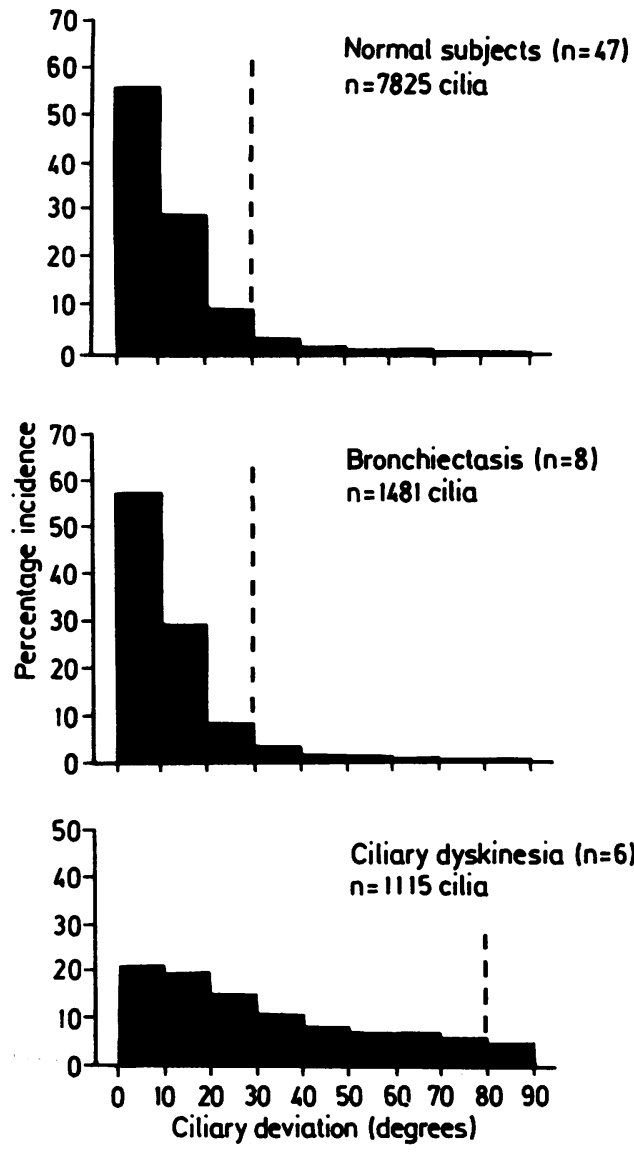

Fig 3 Distribution histograms showing pooled distributions of ciliary angles in normal subjects, patients with bronchiectasis, and patients with ciliary dyskinesia. Dotted lines represent $95 \%$ confidence limits. who had relatively large ciliary deviations the coefficients of variation were smaller than those found for normal subjects (cases 1 and 2). There was a high level of repeatability between observers with all measurements of ciliary deviation in each field and for each patient, being within $5^{\circ}$ of each other. There were no significant differences between the mean ciliary deviation obtained for each patient by the three observers $(p>0.05)$.

\section{INTRAOBERSER VER VARIABILITY}

Table 2 also shows the mean (SD) ciliary deviations, coefficients of variation, and the maximum differences among ciliary deviations obtained for the triplicate copies of each of five fields analysed. Repeated digitising of the same set of lines on micrographs yielded coefficients of variation ranging from $0.7 \%$ to $3.2 \%$, indicating that the major factor that had contributed to the variability of the method was the manual drawing of lines on the micrograph by the observer.

\section{Discussion}

Disorientation of cilia has been described in patients with Kartagener's syndrome and other causes of ciliary dyskinesia but there have only been a few studies where ciliary orientation has been quantified. ${ }^{9-11}$ The orientation of cilia varies in normal subjects but in some patients with respiratory tract disease ciliary orientation may be more disorganised, even to the point of being random. The lack of data on the variations of ciliary orientation in normal subjects has limited the interpretation of data from patients with ciliary dyskinesia. Nielsen et al measured the angles between lines drawn through the central tubules of cilia in 15 normal patients and 27 patients with

Table 2 Results of variability studies in measurements of ciliary deviation

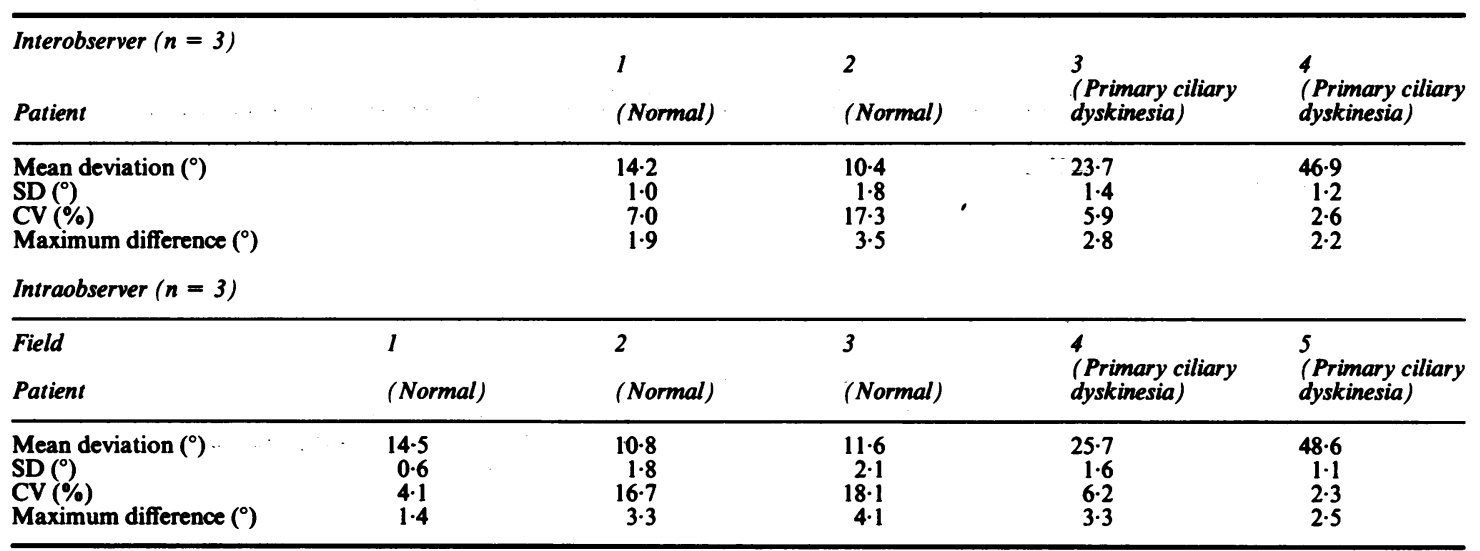

$\mathrm{CV}$, coefficient of variation. 
primary ciliary dyskinesia and found that "ciliary axis" varied between $12^{\circ}$ and $28^{\circ} .9$ Presumably these measurements were made with a protractor. Holley and Afzelius measured manually the orientation of basal feet in eight patients with immotile cilia syndrome and in five normal subjects. " Rautiainen et al also measured the ciliary orientation in primary ciliary dyskinesia and in normal subjects using first a glass angle measure and subsequently an image analysis system, finding similar results with both methods. ${ }^{10}$ is They reported that the ciliary axis in normal subjects varied from $12-41^{\circ} .^{10}$

The method reported here has eliminated much of the time required to measure ciliary angles by hand, is easy to use, and is reproducible. We have used this method in routine screening for ciliary ultrastuctural defects over the past three years. We found that ciliary orientation in our group of normal subjects ranged from $9-25^{\circ}$. These values are similar to those reported by Eliasson et al $^{4}$ and Nielsen et al. ${ }^{9}$ Using a similar methodology to Rautiainen, ${ }^{10}$ we found the variations in ciliary orientation in normal subjects to be lower than reported in his study. This may be because we measured larger numbers of cilia and were able to pool fields within each specimen so that bias from smaller fields or from occasional fields with aberrant cilia was minimised. During the measuring procedure it is important to ensure that all cilia being measured within a field belong to the same contiguous group of cells and do not originate from individual cells or fragments of cells. By only studying cilia from relatively large strips of epithelium and not from aggregations of individual cells the possibility of groups of individual cells producing artefactually high measurements of ciliary orientation is eliminated.

Using our technique ciliary orientation in normal subjects was always less than $30^{\circ}$. Patients with ciliary orientation greater than $30^{\circ}$ all had recurrent respiratory tract disease. Four patients with ciliary orientations greater than $30^{\circ}$ had other ciliary ultrastructural defects, such as defective dynein arms and disorganised tubules, but in one, ciliary disorientation was the only ciliary abnormality found. It is widely recognised that primary ciliary dyskinesia is due to a heterogenous group of defects. ${ }^{16}{ }^{17}$ If ciliary disorientation is not measured such patients may not be detected. Patients with gross ciliary disorientation may have a normal or near-normal ciliary beat frequency, but such patients would still be expected to have impaired mucociliary clearance because the direction of beating is not consistent.

It has been suggested that measurements of ciliary orientation involving the central tubules may be open to error because the central tubules may rotate within the axoneme during bending, or cilia may twist during fixation and processing for electron microscopy. ${ }^{911}$
Hence it has been argued that measurements of ciliary orientation may only be made validly at the level of the basal foot. ${ }^{118}$ Our analysis of ciliary orientation at three levels along the length of the cilium, however, indicates that there is very little variation in the mean ciliary orientation along the cilium and that accurate and representative measurements may be made at almost any point along the cilium. This is consistent with the findings of an ultrastructural study using serial sections of human respiratory cilia ${ }^{19}$ which found that the central tubules do not coil around the axis of the cilium. The only advantage of measuring alignment of the basal feet is that the basal feet indicate the direction of the effective stroke, thus providing a range of ciliary orientation between 0-360 degrees instead of $0-180$ degrees. In practice, however, fortuitous thin sections through the basal feet rarely yield transverse profiles of the basal body, including the basal foot projection (fig 1).

Abnormal ciliary orientation is one of the ultrastructural defects of cilia which can cause recurrent respiratory tract disease. Such a defect might arise secondary to respiratory tract infection that is not due to a primary ciliary abnormality. ${ }^{18}$ This has been suggested in patients with asthma ${ }^{20}$ the common cold, ${ }^{10}$ and in experimental bronchitic rats. ${ }^{21}$ If secondary ciliary disorientation is suspected ciliary orientation should be measured at a different time or in a different ciliated area of the body. ${ }^{22}$ We have shown that in seven patients with bronchiectasis not due to primary ciliary disease, nasal ciliary orientation did not differ from that of normal subjects, suggesting that in these patients respiratory tract disease did not cause ciliary disorientation.

Ciliary orientation can be studied easily in nasal mucosal brushings obtained non-invasively. Measurement of ciliary orientation should be included in any programme for examining ciliary ultrastructural defects in patients with recurrent respiratory disease because in certain patients this may be the only defect present.

We gratefully acknowledge the assistance of $\mathrm{Dr} R$ Newland and Mr JR Perkins of the department of anatomical pathology, Concord Hospital, for access to the facilities of the electron microscopy laboratory, and $\mathrm{Mr} \mathrm{D}$ Crosby for computer programming. This work was supported by a research grant from the Department of Veterans' Affairs, Commonwealth of Australia.

\section{Appendix}

Ideally, if the angles are truly randomly distributed and $\mathbf{n}$ approaches infinity, a uniform density function would result such that all values of $\mathrm{x}$ between $\alpha$ and $\beta$ 
are equally likely (fig 1a) and the probability function of $\mathrm{x}$ is described by:

$$
f(x)=\frac{1}{\beta-\alpha} \text { for } \alpha<x<\beta
$$

From the definition of the mean for a continuous distribution ${ }^{23}$ the mean $(\mu)$ for such a uniform distribution can be calculated by substitution of $f(x)$ into the integral;

$$
\begin{aligned}
\mu & =\int_{\alpha}^{\beta} \mathrm{x} \cdot \mathrm{f}(\mathrm{x}) \cdot \mathrm{d} \mathrm{x} \\
& =\int_{\alpha}^{\beta} \mathrm{x} \cdot \frac{1}{\beta-\alpha} \cdot \mathrm{dx} \\
& =\frac{\alpha+\beta}{2}
\end{aligned}
$$

For $\alpha=-90, \beta=90$;

$\mu=\frac{-90+90}{2}$

$$
=0
$$

Similarly, the variance is defined by the integral;

$$
\begin{aligned}
\mathrm{s}^{2} & =\int_{\alpha}^{\beta} \mathrm{x}^{2} \cdot \frac{1}{\beta-\alpha} \cdot \mathrm{dx}-\mathrm{u}^{2} \\
& =\int_{\alpha}^{\beta} \mathrm{x}^{2} \cdot \frac{1}{\beta-\alpha} \cdot \mathrm{dx}-\left(\frac{\alpha+\beta}{2}\right)^{2} \\
& =\frac{\alpha^{2}+\alpha \beta+\beta^{2}}{3}-\frac{(\alpha+\beta)^{2}}{4} \\
& =\frac{(\alpha-\beta)^{2}}{12}
\end{aligned}
$$

For $a=-90, \beta=90$

$$
\mathrm{s}=51.96
$$

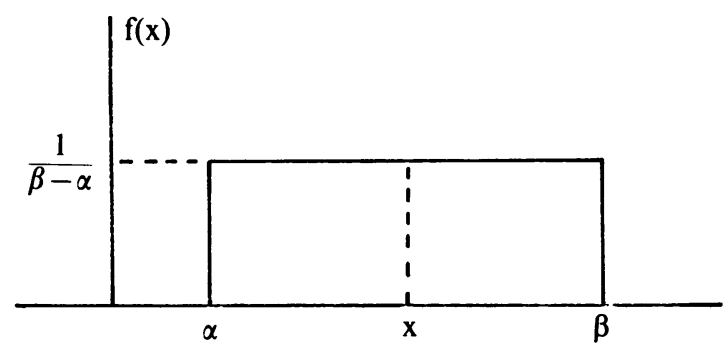

Fig 1A Uniform density distribution of a random population.
Therefore, if a population of ciliary angles is truly random the standard deviation should equal 51.96 degrees.

\section{References}

1 Sade J, Eliezer N, Silberberg A, Nevo AC. The role of mucus in transport by cilia. Am Rev Respir Dis 1970;102:48-52.

2 Anonymous. Pulmonary mucociliary clearance [Editorial]. Lancet 1982;i:203-4.

3 Sleigh MA, Blake JR, Liron N. The propulsion of mucus by cilia. Am Rev Respir Dis 1988;137:726-41.

4 Eliasson R, Mossberg B, Camner P, Afzelius BA. The immotile cilia syndrome. A congenital ciliary abnormality as an etiologic factor in chronic airway infection and male sterility. $N$ Engl $J$ Med 1977;297:1-6.

5 Afzelius BA. A human syndrome caused by immotile cilia. Science 1976;193:317-9.

6 Pedersen H, Mygind N. Absence of axonemal arms in the nasal mucosal cilia in Kartagener's syndrome. Nature 1976;262: 494-5.

7 Sturgess JM, Chao J, Wong J, Aspin N, Turner JAP. Cilia with defective radial spokes: A cause of human respiratory disease. $N$ Engl J Med 1979;300:53-6.

8 Sturgess JM, Chao J, Turner JAP. Transposition of ciliary microtubules: Another cause of impaired ciliary motility. $N$ Engl J Med 1980;303:318-22.

9 Nielsen MH, Pedersen M, Christensen B, Mygind N. Blind quantitative electron microscopy of cilia from patients with primary ciliary dyskinesia and from normal subjects. Eur $J$ Respir Dis 1983;64(Suppl 127):19-30.

10 Rautiainen MEP. Orientation of human respiratory cilia. Eur Respir J 1988;1:257-61.

11 Holley MC, Afzelius BA. Alignment of cilia in immotile-cilia syndrome. Tissue and Cell 1986;18:521-9.

12 Capeder J, Muller W, Dalquen P, Campana A. Kartagenersyndrom. Ein beitrag zur pathogenese und therapie. Dtsch Med Wochenschr 1982;107:1635-9.

13 Rutland J, Cole PJ. Non-invasive sampling of nasal cilia for the measurement of beat frequency and study of ultrastructure. Lancet 1980;ii:564-5.

14 Rutland J, Dewar A, Cox T, Cole PJ. Nasal brushing for the study of ciliary ultrastructure. J Clin Pathol 1982;35:356-9.

15 Rautiainen MEP, Collan Y, Nuutinen J. A method for measuring the orientation ("beat direction") of respiratory cilia. Arch Otorhinolaryngol 1986;243:265-8.

16 Chao J, Turner JAP, Sturgess JM. Genetic heterogeneity of dynein-deficiency in cilia from patients with respiratory disease. Am Rev Respir Dis 1982;126:302-5.

17 Schneeberger EE, McCormack J, Issenberg HJ, Schuster SR, Gerald PS. Heterogeneity of ciliary morphology in the immotile cilia syndrome in man. J Ultrastruct Res 1980;73:34-43.

18 Sleigh MA. Kartagener's syndrome, ciliary defects and ciliary function. Eur J Respir Dis 1983;64(Suppl 127):157-61.

19 Rautiainen MEP, Collan Y, Nuutinen J, Karja J. Ultrastructure of human respiratory cilia. A study based on serial sections. Ultrastruct Pathol 1984;6:331-9.

20 Laitinen LA, Heino M, Laitinen A, Kava T, Haahtela T. Damage of the airway epithelium and bronchial reactivity in patients with asthma. Am Rev Respir Dis 1985;131:599-606.

21 Iravani J, van As A. Mucus transport in the tracheobronchial tree of normal and bronchitic rats. J Pathol 1972;106:81-93.

22 Rutland J, Cox T, Dewar A, Cole P, Warner JO. Transitory ultrastructural abnormalities of cilia. Br J Dis Chest 1982; 76:185-8.

23 Miller I, Freund JE. Probability and statistics for engineers. Englewood Cliffs, New Jersey. Prentice-Hall Inc. 1965:63-80.

Requests for reprints to: Dr J Rutland, Respiratory Unit, Concord Hospital, Hospital Road, Concord, New South Wales, 2139 Australia. 